\title{
Use of a corrugated beam pipe as a passive deflector for bunch length measurements
}

\author{
Jimin Seok and Moses Chung* \\ Department of Physics, Ulsan National Institute of Science and Technology, Ulsan 44919, Korea \\ Heung-Sik Kang, ${ }^{\dagger}$ Chang-Ki Min, and Donghyun Na \\ Pohang Accelerator Laboratory, Pohang 37673, Korea
}

(Received 29 August 2017; published 9 February 2018)

\begin{abstract}
We report the experimental demonstration of bunch length measurements using a corrugated metallic beam pipe as a passive deflector. The corrugated beam pipe has been adopted for reducing longitudinal chirping after the bunch compressors in several XFEL facilities worldwide. In the meantime, there have been attempts to measure the electron bunch's longitudinal current profile using the dipole wakefields generated in the corrugated pipe. Nevertheless, the bunch shape reconstructed from the nonlinearly deflected beam suffers from significant distortion, particularly near the head of the bunch. In this paper, we introduce an iterative process to improve the resolution of the bunch shape reconstruction. The ASTRA and ELEGANT simulations have been performed for pencil beam and cigar beam cases, in order to verify the effectiveness of the reconstruction process. To overcome the undesirable effects of transverse beam spreads, a measurement scheme involving both the corrugated beam pipe and the spectrometer magnet has been employed, both of which do not require a dedicated (and likely very expensive) rf system. A proof-ofprinciple experiment was carried out at Pohang Accelerator Laboratory (PAL) Injector Test Facility (ITF), and its results are discussed together with a comparison with the rf deflector measurement.
\end{abstract}

DOI: 10.1103/PhysRevAccelBeams.21.022801

\section{INTRODUCTION}

Following the theoretical study by Bane and Stupakov [1] on the possibility of using a metallic beam pipe with small periodic corrugations for the energy-chirp control in $\mathrm{x}$-ray free electron lasers (FELs), extensive experimental investigations have been carried out to demonstrate the feasibility of this device (often called the corrugated-wall "dechirper"). The first experimental study on the corrugated dechirper was performed at the PAL-ITF, an electron injector test facility at the Pohang Accelerator Laboratory (PAL) [2]. In this study, the removal of a linear energy correlation in a relativistic electron beam was confirmed experimentally. At the Shanghai deep ultraviolet FEL (SDUV-FEL) facility, on the other hand, the dechirper was applied to linearize the undesired beam energy curvature imprinted by the accelerating rf field [3]. Furthermore, at the Center for Ultrafast Diffraction and

\footnotetext{
*mchung@unist.ac.kr

'hskang@postech.ac.kr
}

Published by the American Physical Society under the terms of the Creative Commons Attribution 4.0 International license. Further distribution of this work must maintain attribution to the author(s) and the published article's title, journal citation, and DOI.
Microscopy at Shanghai Jiao Tong University, it was shown that by using a pair of dechirpers with orthogonal orientations, the quadrupole wakefields could be effectively cancelled, which resulted in a significant reduction in the beam energy spread [4]. At the Accelerator Test Facility at Brookhaven National Laboratory, other types of the dechirper structures have also been proposed and tested, such as the dielectric-lined cylindrical waveguide [5] and the dielectric-lined planar tunable dechirper [6].

The corrugated dechirper is composed of a vacuum chamber consisting of two corrugated, metallic plates with an adjustable gap [2]. Although the flat geometry allows flexibility in changing the gap distance, the quadrupole component of the wakefields becomes very strong as the gap narrows and may degrade the beam emittance $[3,4]$. When the beam has an offset away from the dechirper center, dipole wakefields are generated in addition to the longitudinal and quadrupole wakes. The transverse kick induced in this process makes it possible to use a dechirper as a passive deflector $[7,8]$ or a fast kicker [9]. We also note that the deflecting forces in open accelerating structures $[10,11]$ have similar features as the transverse kicks in the dechirper.

For the bunch length measurement of a short electron beam, the transverse deflecting rf cavity (TCAV) [12] is commonly used to project the longitudinal beam distribution onto a transverse beam profile monitor [12]. The 
TCAV requires a dedicated klystron system in order to control the rf amplitude and phase of the deflecting cavity without altering the neighboring accelerating structures. Therefore, if the dechirper performs effectively as a deflector, it can offer a cost-effective alternative solution for bunch length measurement, replacing the expensive TCAV system. Moreover, there is no need for synchronization in the dechirper setup [8].

One possible problem with using a dechirper as a deflector is that the transverse kicks received by the trailing particles vary nonlinearly with the distance from the bunch head [9]. The head receives essentially no transverse kick, whereas the tail part experiences a much stronger kick. This feature is quite different from the conventional TCAV system, in which the bunch center is usually synchronized to pass through the cavity at the zero-crossing rf phase; thus, the bunch head and tail receive an equal amount of kick in opposite directions [12]. In the TCAV setup, the transverse kick varies linearly between the head and the tail to a good approximation.

Several theoretical investigations have been made to characterize the excitation of the transverse wakes and their self-interaction with the beam bunch itself $[8,9,13]$. Evidence of a strong dipole kick in the off-centered corrugated dechirper was reported in Ref. [2], and the results of a proof-of-principle experiment for a passive deflector concept using a dielectric-lined waveguide were discussed in Ref. [14]. Moreover, recent measurements provide very detailed information on the wakefields and beam deflections in the corrugated dechirper [15-18]. In this paper, we further investigate this novel idea of measuring the bunch length using a dechirper of rectangular geometry (which would provide more operational flexibility [2]), and introduce a method to reconstruct the bunch shape even in the presence of nonlinear transverse kicks. Unlike the reconstruction methods introduced by Novokhatski $\mathrm{et}$ al. [15] and Bettoni et al. [14], our method comprises two stages. First, we apply an iterative reconstruction method for pencil beams, which solves the nonlinearity problems. Then, a measurement scheme involving both the dechirper and spectrometer magnet is employed to handle transverse beam spreads in realistic cigar beams. In Sec. II, theoretical backgrounds are reviewed, and the basic characteristics of the corrugated-wall dechirper are presented. A bunch shape reconstruction method for a pencil beam (i.e., a beam with no transverse dimensions) is introduced in Sec. III together with numerical examples. In Sec. VI, we apply our reconstruction method to the experimental results obtained from the PAL-ITF, and compare them with TCAV measurements. Our conclusions and directions for future work are summarized in Sec. V.

\section{THEORETICAL BACKGROUND}

An ultrarelativistic beam having a non-negligible offset from the dechirper center has short-range transverse wakes between two point charges that are approximately given as

$$
\begin{aligned}
& w_{x}(s)=w_{q}(s)\left(x_{l}-x_{t}\right), \\
& w_{y}(s)=w_{d}(s) y_{l}+w_{q}(s)\left(y_{t}-y_{l}\right),
\end{aligned}
$$

where $x_{l}$ and $y_{l}$ are the offsets of the leading charge, and $x_{t}$ and $y_{t}$ are the offsets of the trailing one [2]. Here, $w_{d}(s)$ and $w_{q}(s)$ are the dipole and quadrupole wake functions, respectively. The $w_{d}(s)$ and $w_{q}(s)$ functions have a dimension of $\mathrm{V} /\left(\mathrm{Cm}^{2}\right)$ (i.e., we assume the wake is distributed along an extended path [19]), and they are dependent on the gap as $\propto a^{-4}$ [2]. The leading charge moves according to $z_{l}=c t$, and the trailing charge follows the leading charge at a distance $s$ behind (for $s>0$ ) according to $z_{t}=c t-s$. For the region ahead of the wake source $s<0, w_{d}(s)=w_{q}(s)=0$ because of the causality principle. If the beam offset increases and becomes comparable to the size of the gap, then the assumption that the dipole wake is linear in $y_{l}$ breaks down [9]. In this case, we might need higher order analytical formulas or numerical calculations. For the experimental settings employed in this feasibility study, the offset value of $2 \mathrm{~mm}$ is still sufficiently small compared to the gap distance $2 a=8 \mathrm{~mm}$. Therefore, the linearity in $w_{d}(s) y_{l}$ is still a good approximation to estimate the vertical kicks received by the beam tail (see, for example, Fig. 7 of Ref. [2]). This linear approximation also simplifies the iteration process during the bunch shape reconstruction.

For an arbitrary current distribution, the voltage in the $y$-direction (per $1 \mathrm{~m}$ of a chamber length and $1 \mathrm{C}$ of charge) is then [20]

$$
V_{y}(\tau)=\int_{-\infty}^{\tau} w_{y}\left[c\left(\tau-\tau^{\prime}\right)\right] I\left(\tau^{\prime}\right) d \tau^{\prime},
$$

where $I(\tau)$ is the normalized current distribution in the time domain, i.e., $\int_{-\infty}^{\infty} I\left(\tau^{\prime}\right) d \tau^{\prime}=1$, and $c \tau=z_{0}-z$ is the particle position with respect to the reference particle. The normalized current distribution can also be expressed as $I(\tau)=\lambda(\tau) c / Q_{\text {tot }} \geq 0$, where $Q_{\text {tot }}=\int_{-\infty}^{\infty} \lambda\left(\tau^{\prime}\right) c d \tau^{\prime}$ is the total bunch charge, and $\lambda$ is the line charge density. For a pencil beam, we have $y_{t} \approx y_{l}$ and

$$
V_{y}(\tau) \approx y_{l} \int_{-\infty}^{\tau} w_{d}\left[c\left(\tau-\tau^{\prime}\right)\right] I\left(\tau^{\prime}\right) d \tau^{\prime}
$$

The dipole wake function $w_{d}$ is positive along the bunch [9]. Therefore, for a positive offset $y_{l}>0$ the tail part receives positive momentum kicks, whereas for a negative offset $y_{l}<0$, the tail part receives negative momentum kicks. This feature is independent of the sign of the beam charge. The transverse momentum kick for a beam particle with charge $q$ is then 


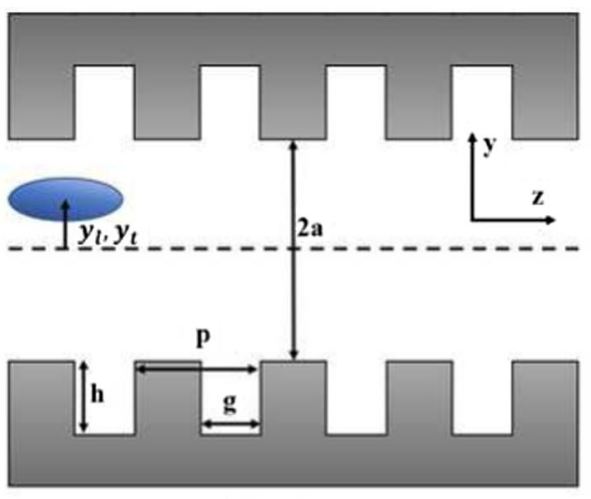

Side view

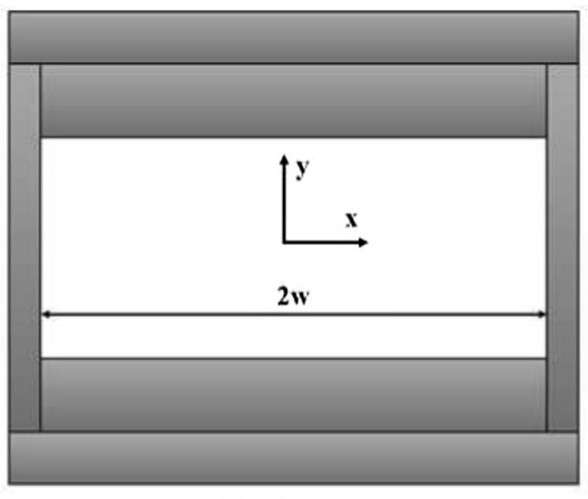

Front view

FIG. 1. Geometry of the corrugated-wall dechirper [1,2]. The blue ellipsoid indicates a beam bunch moving parallel to the $z$-axis with a transverse offset.

$$
c \Delta p_{y}(\tau)=q Q_{\mathrm{tot}} V_{y}(\tau) L
$$

where $L$ is the length of the corrugated chamber. Note that the unit of $c \Delta p_{y}$ is coulomb - volt as desired.

If the charge in the bunch has an initial longitudinal momentum $p_{z} \approx E_{0} / c$, then the charge will have a trajectory with angle [21]

$$
\Delta y^{\prime}=\tan ^{-1}\left(\frac{\Delta p_{y}}{p_{z}}\right) \approx \frac{c \Delta p_{y}}{E_{0}},
$$

where $E_{0}$ is the reference energy of the beam bunch. As mentioned earlier, the head receives essentially no transverse kick, whereas the tail part experiences a stronger vertical deflection.

Figure 1 shows the structure of the corrugated-wall dechirper. There are two corrugated metallic plates in parallel. Each plate is equipped with an independent motorized linear translation stage so that the gap distance

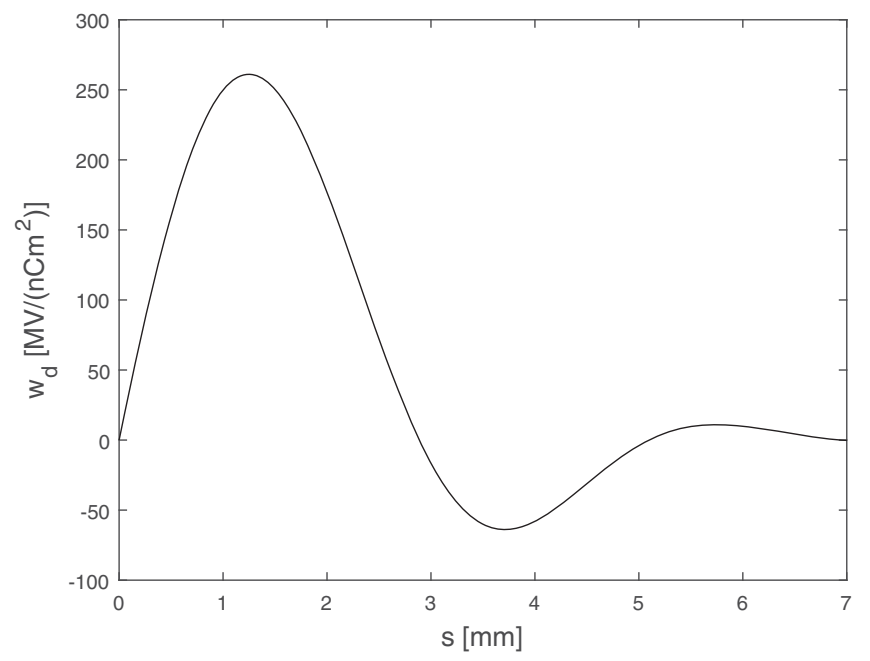

FIG. 2. The dipole wakefields calculated with the analytical model. (2a) and the beam offset $\left(y_{l}\right.$ or $\left.y_{t}\right)$ can be changed independently. The gap between the two plates $2 a$ can be adjusted from $5 \mathrm{~mm}$ to $28 \mathrm{~mm}$. The geometric parameters of the dechirper are the corrugation period $p=0.5 \mathrm{~mm}$, corrugation height $h=0.6 \mathrm{~mm}$, corrugation gap $g=0.3 \mathrm{~mm}$, and plate width $2 w=50 \mathrm{~mm}$. The corrugated structure is made of aluminum, and the axial length is $L=1 \mathrm{~m}$. With these parameters $(p, h, g \ll 2 a)$, we calculate the wake functions in the corrugated-wall dechirper using the analytical models in Refs. [22,23]. We assume a steeply corrugated structure (i.e., $h \geq p$ ) and neglect the transient effect by noting that the catch-up distance is much shorter than the dechirper length [1]. The dipole wakefields used for the reconstruction of the bunch charge distribution are shown in Fig. 2.

\section{RECONSTRUCTION METHOD}

Our goal is to extract information on the longitudinal current distribution from the transverse beam profile measured on the screen. The nonlinearity in the vertical deflection makes this reconstruction process quite challenging, particularly when the image is blurred along the deflecting direction (i.e., the $y$-direction in this study). In other words, each single point for a given $y$ position on the screen contains information before and after that point in the longitudinal direction. Such blurring effects are due to the finite transverse emittance of the beam, quadrupole kicks from the dechirper, and limited resolution of the screen and imaging system. To tackle this problem, we first consider a pencil beam (or a thin beam) in which the beam has no transverse excursions. Later, we apply the reconstruction method developed for a pencil beam to a cigar beam (or a fat beam) in which the beam has finite transverse dimensions.

\section{A. Pencil (thin) beam case}

When a pencil beam is propagating through the dechirper with a transverse offset, the quadrupole 
wakefields do not need to be considered, and the calculation of the transverse momentum kick becomes much simpler [see Eqs. (4) and (5)]. The proposed reconstruction method has four steps, and the iteration process continues until its output converges.

The first step is to calculate the voltage gain given in Eq. (4), which requires a normalized current distribution $I(\tau)$. However, the current distribution $I(\tau)$ is initially unknown; therefore, we start the iteration process with a guessed distribution $I_{\text {guess }}(\tau)$ :

$$
V_{y}(\tau) \approx y_{l} \int_{-\infty}^{\tau} w_{d}\left[c\left(\tau-\tau^{\prime}\right)\right] I_{\text {guess }}\left(\tau^{\prime}\right) d \tau^{\prime}
$$

For the very first iteration, we pick the normalized Gaussian distribution for $I_{\text {guess }}(\tau)$, and for the rest of the process, $I(\tau)$ obtained from the previous iteration cycle is assigned to $I_{\text {guess }}(\tau)$.

The second step is to calculate the vertical position of the particle $y$ on the screen (either screen-5 or screen- 6 in Fig. 3):

$$
y \approx y_{0}+R_{34} \Delta y^{\prime}=y_{0}+R_{34}\left[\frac{q Q_{\mathrm{tot}} V_{y}(\tau) L}{E_{0}}\right],
$$

where $R_{34}$ is the $y-y^{\prime}$ component of the transfer matrix from the dechirper to the screen [12], and $y_{0}$ is the particle position on the screen without any deflection. The approximation in Eq. (8) is valid because the beam is assumed to be very thin.

The third step is to update the new normalized current distribution $I_{\text {new }}(\tau)$ using the $y$-directional normalized intensity distribution $f(y)$ on the screen. $f(y)$, the only data available during the experiment, contains information on the current distribution. Because both $f(y)$ and $I_{\text {new }}(\tau)$ are normalized functions, we have

$$
\int_{-\infty}^{\infty} f(y) d y=\int_{-\infty}^{\infty} I_{\text {new }}(\tau) d \tau=1
$$

Furthermore, if the transformation from $\tau$ to $y$ is monotonic (i.e., a one-to-one mapping), it then follows that

$$
I_{\text {new }}(\tau)=f(y)\left|\frac{d y}{d \tau}\right| .
$$

If the bunch length is too long, the dipole wake may decrease at some point, when $V_{y}(\tau)$ would no longer be monotonic. In this case, the mapping has a two-to-one nature. One could handle this two-to-one mapping by dividing the $\tau$ coordinates into two intervals, in such a way that at each interval the mapping $y(\tau)$ becomes one to one. Nevertheless, this additional step would make the reconstruction process much more complicated, and hence in this study we only consider the case where the bunch is short enough that the momentum kick $\Delta p_{y}$ is always monotonic.

The final step is to adjust $I_{\text {guess }}(\tau)$ in Eq. (7) through

$$
I_{\text {guess }}(\tau) \rightarrow I_{\text {guess }}(\tau)+\alpha\left[I_{\text {new }}(\tau)-I_{\text {guess }}(\tau)\right] \text {, }
$$

where $\alpha$ is the relaxation factor that controls the convergence of the iteration process. We repeat this four-step iteration process until the difference between $I_{\text {new }}(\tau)$ and $I_{\text {guess }}(\tau)$ becomes sufficiently small over the length of the beam bunch.

To verify the effectiveness of the proposed reconstruction method before we apply it to the actual experimental data, we perform numerical simulations. Two simulation tools, ASTRA [25] and ELEGANT [26], were used. The simulations are carried out with the lattice settings depicted in Fig. 3 and Table I. Up to the accelerating cavity $\mathrm{LOb}$, an electron beam is generated and transported by ASTRA, and after LOb, ELEGANT calculates the downstream beam transport including the beam-wake interaction in the dechirper. In Fig. 4, the full width at half maximum (FWHM) of the input beam at the dechirper is 3.59 ps. Making use of the pencil beam's intensity distribution $f(y)$ obtained on screen-6 [Fig. 4(a)], we reconstruct the current distribution $I(\tau)$ on the basis of the proposed iterative method. The FWHM of the reconstructed current distribution turns out to be $3.53 \mathrm{ps}$, which matches the input value well. The overall shapes of the two current distributions in Fig. 4(b) are in good agreement with each other, although there are some discrepancies pertaining to the spikes in the distributions. Such spikes are associated with numerical

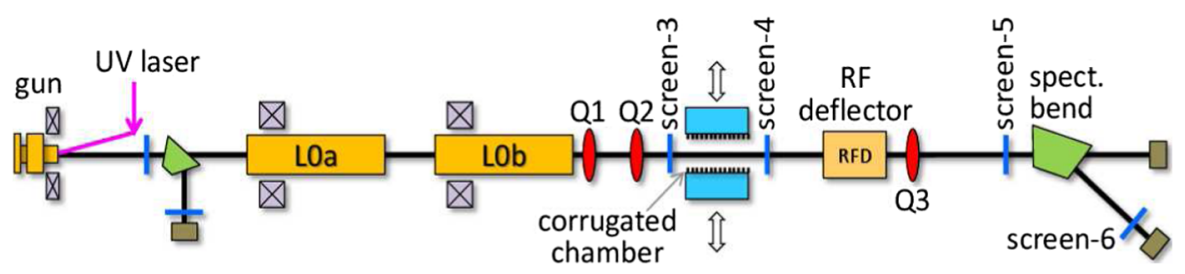

FIG. 3. Layout of the PAL-ITF beam line [2,24]. Two accelerating cavities LOa and LOb are available in the beam line. For this passive deflector experiment, however, L0b was turned off. The corrugated-wall chamber is located between the positions of screen-3 and screen-4, and the RF deflecting cavity is installed after screen-4. Before the spectrometer magnet, screen-5 is inserted, and screen-6 measures the spectrometer output. 
TABLE I. Summary of the ITF system and typical beam parameters used for this experiment.

\begin{tabular}{lllc}
\hline \hline Component & \multicolumn{1}{c}{ Parameter } & Value & Unit \\
\hline \multirow{4}{*}{ rf gun } & Operating frequency & 2.856 & $\mathrm{GHz}$ \\
& rf-pulse width & 2 & $\mu \mathrm{s}$ \\
Laser & Repetition rate & 10 & $\mathrm{~Hz}$ \\
\multirow{5}{*}{ Electron beam } & FWHM & 3 & $\mathrm{ps}$ \\
& Wavelength & 253 & $\mathrm{~nm}$ \\
& Energy & $\sim 70$ & $\mathrm{MeV}$ \\
& Charge & $\sim 200$ & $\mathrm{pC}$ \\
& Energy spread (rms) & 0.1 & $\%$ \\
& Transverse beam size $\left(\sigma_{x}, \sigma_{y}\right)$ & 0.6 & $\mathrm{~mm}$ \\
& Normalized emittance & $0.5-1$ & $\mu \mathrm{m}$ \\
\hline \hline
\end{tabular}

noise (e.g., due to the finite number of simulation particles), and will not be a problem when the input current distribution is made sufficiently smooth. The convergence properties of the iterative method have been illustrated in Ref. [13], where the average relative error in the current distribution reconstruction became less than $1 \%$ after 16 iteration cycles.

\section{B. Cigar (fat) beam case}

As mentioned previously, a realistic beam has finite transverse excursions, which invalidates the assumption of a pencil beam. Indeed, a cigar (fat) beam's intensity distribution $F(y)$ on the screen is the convolution of the functions $f(y)$ and $S(y, \Delta y)$, which is given by

$$
F(y)=\int_{-\infty}^{\infty} f\left(y^{\prime}\right) S\left(y^{\prime}, y-y^{\prime}\right) d y^{\prime},
$$

where $f(y)$ is the pencil beam's intensity distribution, and $S(y, \Delta y)$ is a spread function. Note that if $S\left(y^{\prime}, y-y^{\prime}\right)=\delta\left(y-y^{\prime}\right)$, then $F(y)=f(y)$. To extract $f(y)$ directly from $F(y)$ measured on screen-5 (i.e., before the spectrometer magnet), we need detailed information on the spread function. One could estimate $S(y, \Delta y)$ by first measuring the transverse beam size $\sigma_{y}=\sqrt{\left\langle y^{2}\right\rangle}$ on screen-5 without the dechirper. For example, one may consider $S(y, \Delta y) \sim 1 /\left(\sigma_{y} \sqrt{2 \pi}\right) \exp \left[-\Delta y^{2} / 2 \sigma_{y}^{2}\right]$. However, in this case, we cannot resolve the variation of the spread function along $y$ and lose information on the quadrupole wake and bunch shape. Some of the initial studies indicate that the reconstruction based on the simple $y$-independent spread function was not successful [13].

On the other hand, if a dechirper is equipped with a spectrometer magnet as in the PAL-ITF (see Fig. 3), we can effectively estimate the spread function $S(y, \Delta y)$ and perform deconvolution to extract $f(y)$. First, we assume that the energy distribution of the cigar beam is approximately the same as that of the corresponding pencil beam. Furthermore, we assume that the energy spread for a given
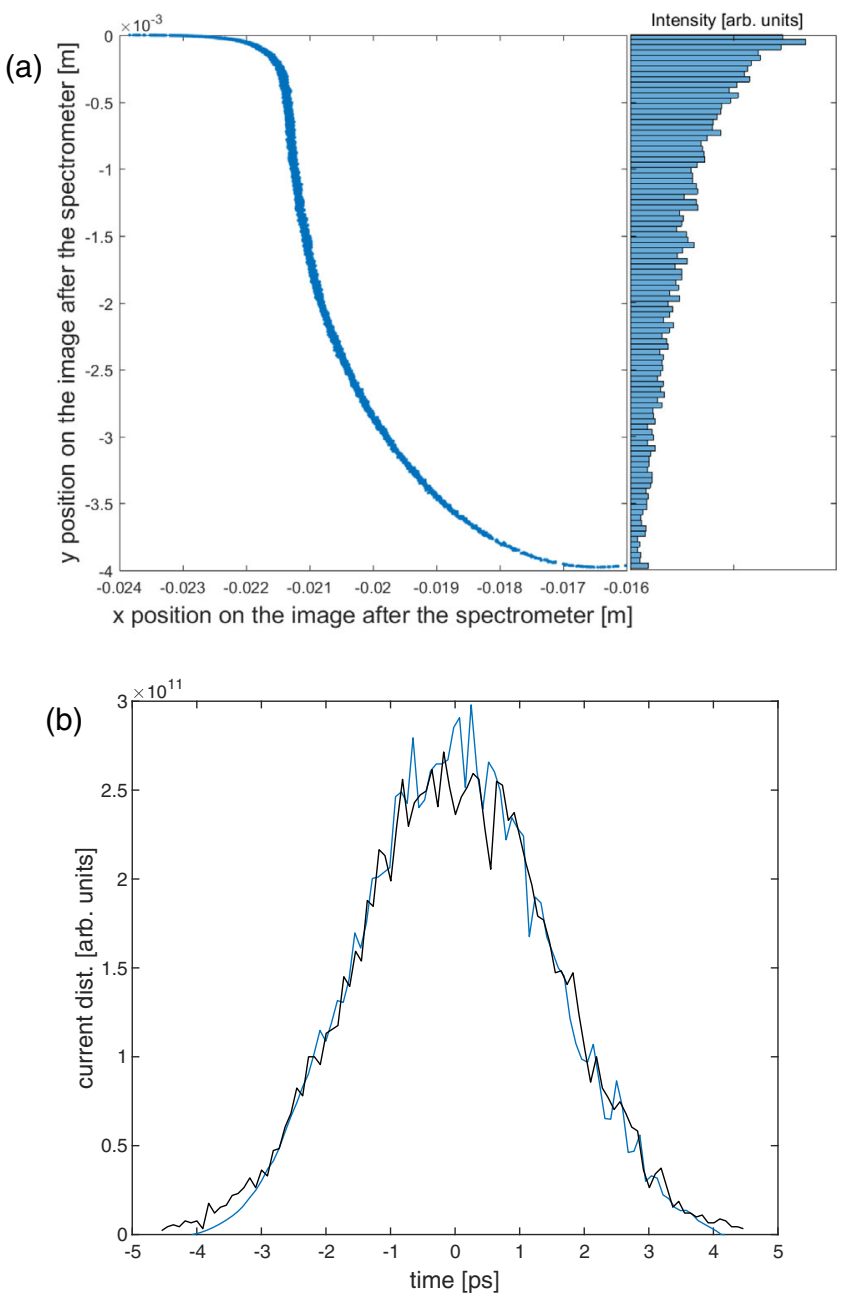

FIG. 4. (a) Simulation results of the two-dimensional particle distribution and intensity distribution $f(y)$ of the pencil beam on screen-6, and (b) corresponding current distributions. The blue line in (b) represents the input current distribution at the dechirper and the black line the reconstructed current distribution.

longitudinal position $(\tau)$ is small compared to the overall energy spread of the bunch. With these conditions, the intensity distribution along the $y$-direction for a given horizontal position $x$ on screen-6 [see Fig. 5(a)] is a good approximation of $S(y, \Delta y)$. Each central point of the spread function $S(y, \Delta y)$ comprises the projection of the pencil beam [see black diamonds in Fig. 5(a)]. Finally, the deconvoluted intensity distribution $f(y)$ is shown in Fig. 5(b).

Once $f(y)$ is obtained, we can apply the proposed reconstruction method to extract the longitudinal current distribution $I(\tau)$. Figure 6 shows the current distributions of a cigar beam. The FWHMs of the input beam (blue line in Fig. 6) and the reconstructed beam (black line in Fig. 6) are $3.6 \mathrm{ps}$ and $3.5 \mathrm{ps}$, respectively. The overall shapes of the two current distributions in Fig. 6 are in good agreement with each other except for the short-time-scale $(\sim 0.1 \mathrm{ps})$ current spikes. This implies that the resolution of the 


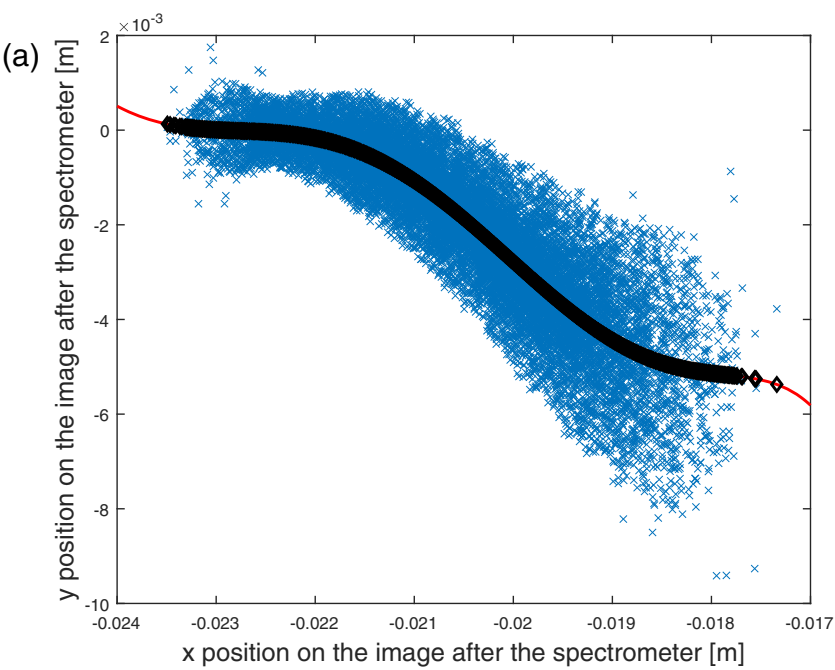

(b)

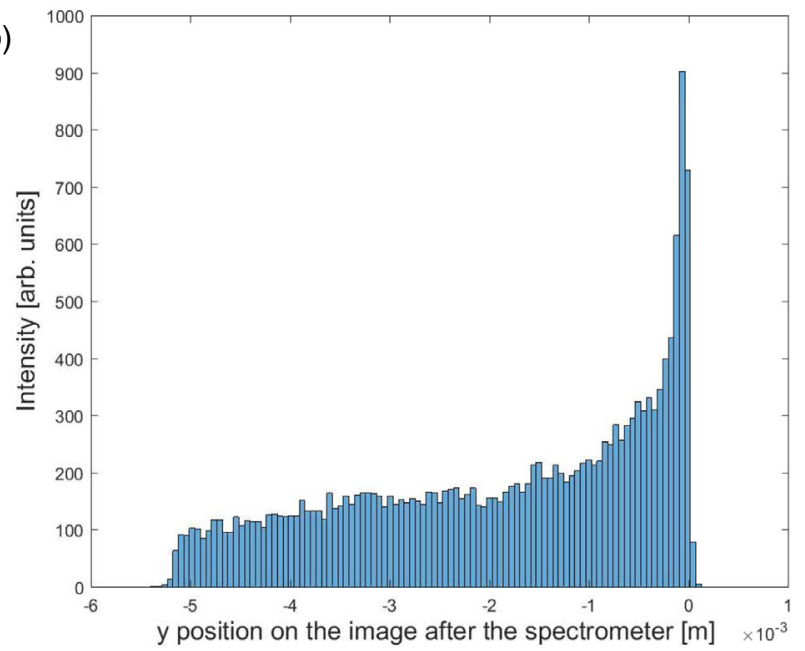

FIG. 5. (a) Simulation results of the two-dimensional particle distribution projected on screen-6, and (b) the corresponding pencil beam's intensity distribution $f(y)$. The black diamonds with red curve in (a) represent the projection of the pencil beam.

present bunch shape measurement technique would be no better than $\sim 0.1 \mathrm{ps}$. We note, on the other hand, that the active deflectors based on X-band klystron system have reached approximately femtosecond-level temporal resolutions [27,28].

\section{EXPERIMENTAL RESULTS}

In Sec. III, the validity of the proposed reconstruction method has been confirmed through numerical simulations for both the pencil beam and cigar beam cases. In this section, we apply our reconstruction method to the experimental results obtained at the PAL-ITF facility. The beam line setting is illustrated in Fig. 3. The ITF consists of a photocathode rf gun, accelerating structures, two sets of klystron-modulator systems, and various instruments (screens, spectrometer magnets, current transformer etc.)

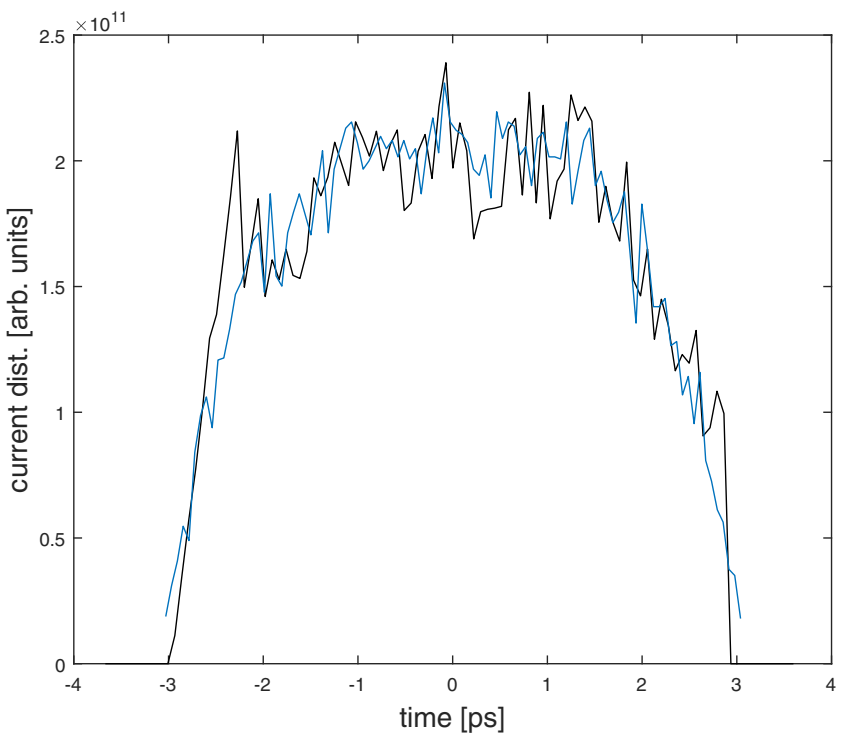

FIG. 6. Simulation results of the current distributions for a cigar (fat) beam. The blue line represents the input current distribution at the dechirper and the black line the reconstructed current distribution.

[24,29]. A 30-mJ Ti:Sa laser system provides a UV laser pulse for the photocathode rf gun. In this experiment, we set the laser pulse width to be 3 ps FWHM. The second linac cavity $\mathrm{LOb}$ and all the quadrupole magnets were turned off for this experiment. The effects of the reference beam energy and transverse focusing on the bunch shape reconstruction will be investigated later in future work. Under these conditions, the nominal electron beam charge and energy are $200 \mathrm{pC}$ and $70 \mathrm{MeV}$, respectively. The transverse beam profiles are measured using two YAG scintillator screens (indicated by screen- 5 and screen- 6 in Fig. 3), which are imaged by CCD cameras. The dechirper has a half gap of $4 \mathrm{~mm}$ (i.e., $2 a=8 \mathrm{~mm}$ ) and an offset of $2 \mathrm{~mm}$ (i.e., $y_{l}=y_{t}=2 \mathrm{~mm}$ ).

Figure 7 shows the screen- 6 image obtained in the experiment. As expected, the real beam exhibit a feature of a cigar (fat) beam, with a finite spread along the vertical direction. Applying the reconstruction strategy introduced in Sec. III, we first extract the corresponding pencil beam from the cigar beam's distribution on the screen- 6 image [see Fig. 7(b)]. The noisy fluctuations before and after the relatively smooth part of the black curve in Fig. 7(b) are caused by the residual background noises (or dead pixels) in the image. We cut off these noisy parts and use only the smooth part for further data processing. The intensity distribution $f(y)$ of the corresponding pencil beam extracted from the screen-6 image is illustrated in Fig. 8.

Finally, we apply our iterative reconstruction method and obtain the longitudinal current profile $I(\tau)$ as shown in Fig. 9 (black line). We also compare our reconstruction output with the measurement result obtained by the conventional transverse deflecting rf cavity (TCAV) technique. 
(a)

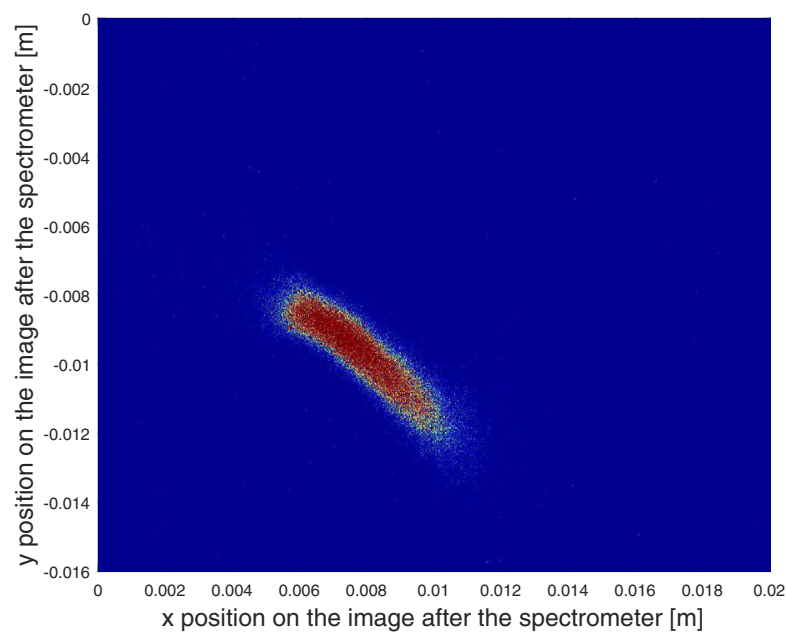

(b)

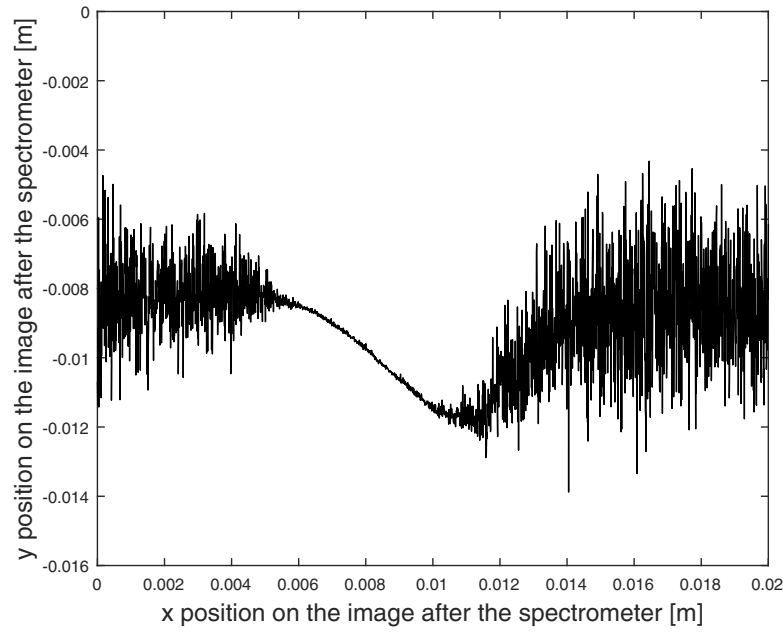

FIG. 7. (a) Screen-6 image taken with dechirper offset of $2 \mathrm{~mm}$ and spectrometer magnet on, and (b) the projected image of the corresponding pencil beam. The bunch head is at the upper-left corner of the screen- 6 image.

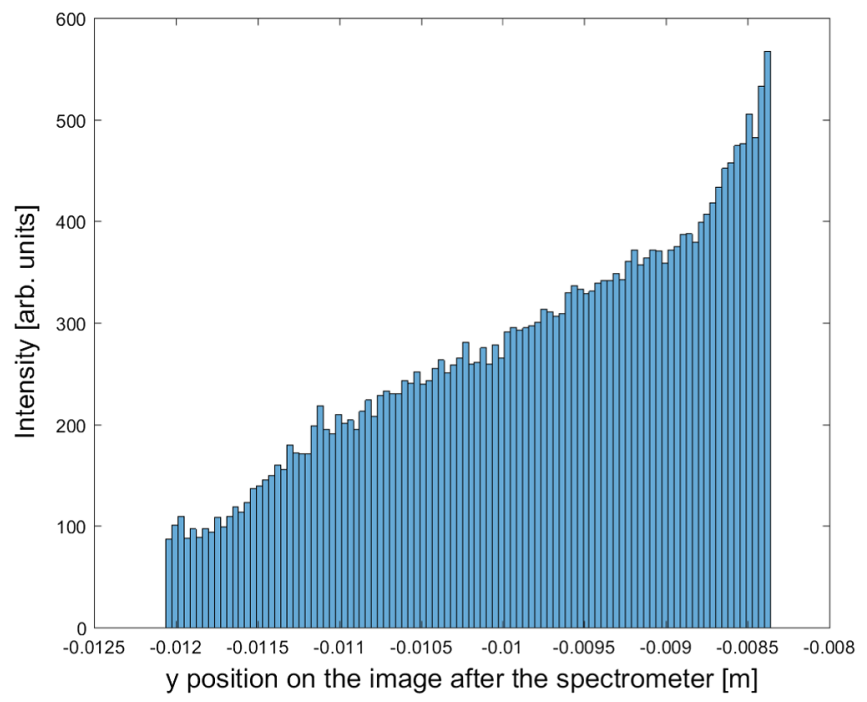

FIG. 8. Intensity distribution $f(y)$ of the pencil beam extracted from the screen-6 image.

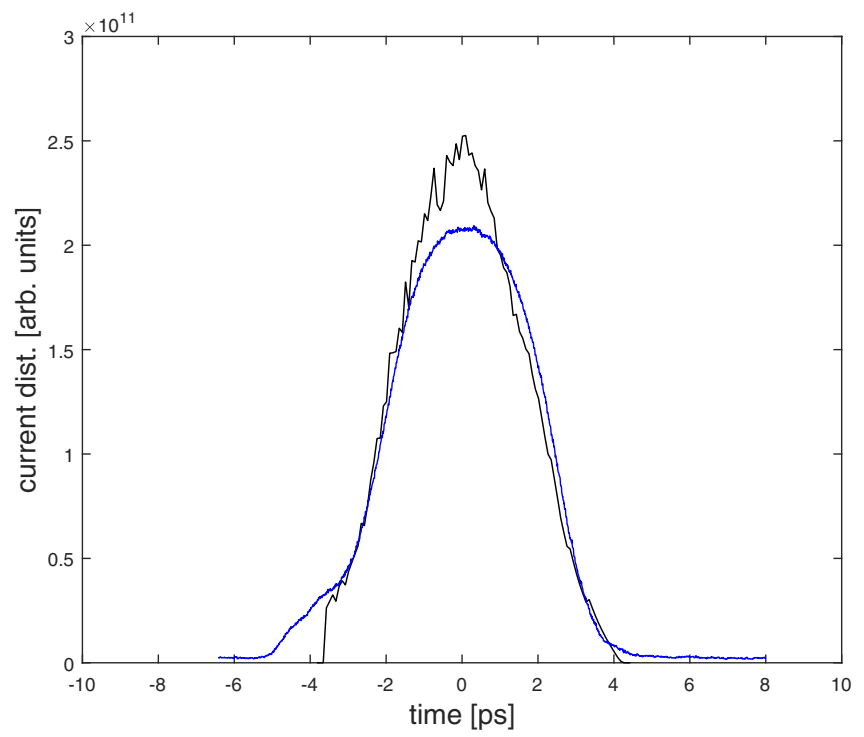

FIG. 9. Longitudinal current profiles $I(\tau)$ are measured either by the dechirper (black line) or by the TCAV (blue line). The measured FWHMs are $3.895 \pm 0.058$ ps (black line) and $4.265 \pm 0.021 \mathrm{ps}$ (blue line), respectively. The negative time $(\tau<0)$ corresponds to the front part of the bunch.

The TCAV (S-band, 10-fs resolution) is installed between the dechirper and the spectrometer magnet (see Fig. 3), which allows longitudinal current profile measurements to be made using screen-5 and enables comparisons with the dechirper data. Figure 10 presents the screen- 5 image taken with the TCAV on and the corresponding intensity distribution along the vertical direction. We note that the intensity distribution $F(y)$ in Fig. 10 is rather symmetric about its center, which is not the case for $f(y)$ in Fig. 8 . This illustrates a quite different feature of the transverse kicks between the TCAV and the dechirper. Figure 9 compares the longitudinal profiles measured by the dechirper (black line) and the TCAV (blue line). The FWHMs of each profile are $3.895 \pm 0.058 \mathrm{ps}$ from the dechirper and $4.265 \pm 0.021 \mathrm{ps}$ from the TCAV, respectively. We note that the laser pulse width for the $\mathrm{rf}$ gun is set to 3 ps FWHM for both cases. During the extraction of the pencil beam from the screen- 6 image data, we cut off the noisy fluctuations in the head and tail parts of $f(y)$, which might account for one of the reasons why the FWHM measured from the dechirper is slightly $(\sim 10 \%)$ shorter. Further, in this analysis we did not apply a deconvolution to $F(y)$ of the TCAV data, which requires an assumption regarding the spread function. The overall shapes of the two profiles are in reasonably good agreement, which demonstrates the effectiveness of the dechirper as a passive deflector for bunch length measurements.

\section{CONCLUSIONS AND FUTURE WORK}

In this paper, we report the results of a proof-of-principle experiment on the use of a corrugated beam pipe 


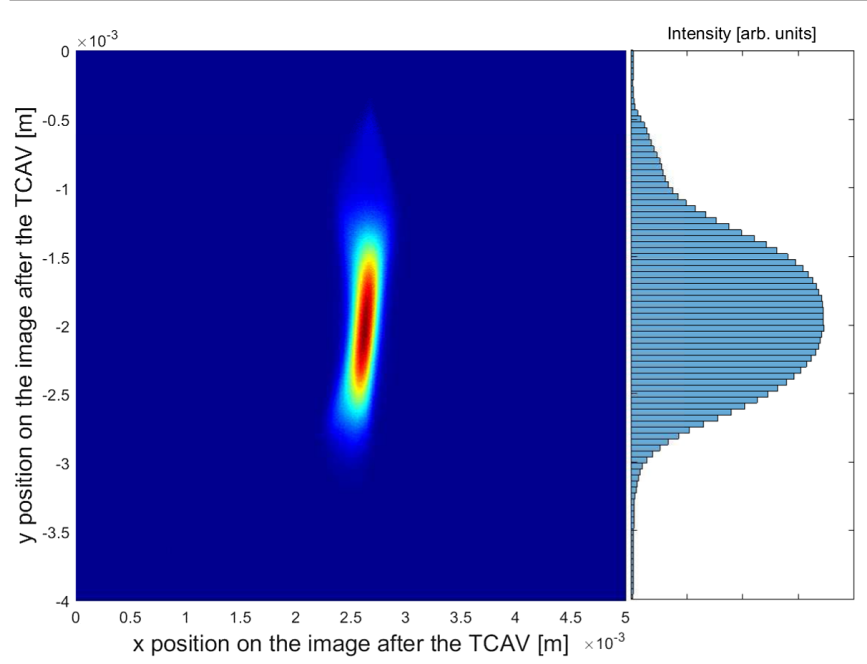

FIG. 10. Screen-5 image taken with transverse deflecting cavity on, and the corresponding intensity distribution $F(y)$ along the vertical direction.

(i.e., dechirper) as a passive deflector for bunch length measurements. We identify the factors that might limit the streaking performance of the dechirper, such as the nonlinearity in the dipole wakefield kick and the effects of the transverse beam spread. To overcome these unfavorable features, we first suggest an iterative reconstruction method for pencil beams, which solves the nonlinearity problems. To handle the transverse beam spreads, a measurement scheme involving both the dechirper and spectrometer magnet has been employed, which enables the corresponding pencil beam to be extracted from the screen image of a real cigar beam. A comparison of the longitudinal current profiles obtained by the dechirper and TCAV measurements reveals reasonably good agreement (less than $10 \%$ of relative differences in FWHMs for five repeated measurements). Hence, we confirmed that the dechirper could be used as a deflector, but with a much simpler hardware setup (i.e., no expensive dedicated rf system and no rf jitter/synchronization issues).

To further improve the resolution of our reconstruction method, we plan to apply transverse focusing by quadrupoles, which will squeeze the transverse dimensions of the beam before it enters the dechirper. Moreover, we consider using a pair of dechirpers with orthogonal orientations to eliminate the effects of the quadrupole wakes. If the transverse effects can be sufficiently minimized through these steps, the bunch length could be measured with the dechirper only, without the need for any ad hoc deconvolution process using the spectrometer. The ultimate resolution and sensitivity of our reconstruction method will be further characterized by means of a series of numerical simulations. In addition, the possibility of applying the dechirper to measure more complex longitudinal current profiles (e.g., beam profiles with multiple current peaks and microstructure [29]) will be investigated in detail.

\section{ACKNOWLEDGMENTS}

This work was supported by the National Research Foundation of Korea (Grants No. NRF-2015R1D1A1A01061074 and No. NRF-2016R1A5A1013277) funded by the Korean government. This work was also supported by the Pohang Accelerator Laboratory (PAL) XFEL project.

[1] K. L. F. Bane and G. Stupakov, Corrugated pipe as a beam dechirper, Nucl. Instrum. Methods Phys. Res., Sect. A 690, 106 (2012).

[2] P. Emma, M. Venturini, K. L. F. Bane, G. Stupakov, H.-S. Kang, M. S. Chae, J. Hong, C.-K. Min, H. Yang, T. Ha et al., Experimental Demonstration of Energy-Chirp Control in Relativistic Electron Bunches Using a Corrugated Pipe, Phys. Rev. Lett. 112, 034801 (2014).

[3] H. Deng, M. Zhang, C. Feng, T. Zhang, X. Wang, T. Lan, L. Feng, W. Zhang, X. Liu, H. Yao et al., Experimental Demonstration of Longitudinal Beam Phase-Space Linearizer in a Free-Electron Laser Facility by Corrugated Structures, Phys. Rev. Lett. 113, 254802 (2014).

[4] F. Fu, R. Wang, P. Zhu, L. Zhao, T. Jiang, C. Lu, S. Liu, L. Shi, L. Yan, H. Deng et al., Demonstration of Nonlinear-EnergySpread Compensation in Relativistic Electron Bunches with Corrugated Structures, Phys. Rev. Lett. 114, 114801 (2015).

[5] S. Antipov, C. Jing, M. Fedurin, W. Gai, A. Kanareykin, K. Kusche, P. Schoessow, V. Yakimenko, and A. Zholents, Experimental Observation of Energy Modulation in Electron Beams Passing through Terahertz Dielectric Wakefield Structures, Phys. Rev. Lett. 108, 144801 (2012).

[6] S. Antipov, S. Baturin, C. Jing, M. Fedurin, A. Kanareykin, C. Swinson, P. Schoessow, W. Gai, and A. Zholents, Experimental Demonstration of Energy-Chirp Compensation by a Tunable Dielectric-Based Structure, Phys. Rev. Lett. 112, 114801 (2014).

[7] V. A. Dolgashev, P. Emma, M. D. Forno, A. Novokhatski, and S. Weathersby, Attosecond Diagnostics of Muti-GeV Electron Beams Using W-Band Deflectors, in the Workshop on Femto-second Electron Imaging and Spectroscopy (FEIS-2), East Lansing, MI, 2015 (unpublished), http:// www.bt.pa.msu.edu/FEIS-2/talks/FEIS-2_Dolgashev.pdf.

[8] A. Novokhatski, Wakefield potentials of corrugated structures, Phys. Rev. ST Accel. Beams 18, 104402 (2015).

[9] K. Bane, G. Stupakov, and I. Zagorodnov, Analytical formulas for short bunch wakes in a flat dechirper, Phys. Rev. Accel. Beams 19, 084401 (2016).

[10] M. Dal Forno, V. Dolgashev, G. Bowden, C. Clarke, M. Hogan, D. McCormick, A. Novokhatski, B. Spataro, S. Weathersby, and S. G. Tantawi, Experimental measurements of rf breakdowns and deflecting gradients in mmwave metallic accelerating structures, Phys. Rev. Accel. Beams 19, 051302 (2016).

[11] M. Dal Forno, V. Dolgashev, G. Bowden, C. Clarke, M. Hogan, D. McCormick, A. Novokhatski, B. O'Shea, B. Spataro, S. Weathersby et al., rf breakdown measurements in electron beam driven $200 \mathrm{GHz}$ copper and copper-silver accelerating structures, Phys. Rev. Accel. Beams 19, 111301 (2016). 
[12] R. Akre, L. Bentson, P. Emma, and P. Krejcik, in Proceedings of the 19th Particle Accelerator Conference, Chicago, IL, 2001 (IEEE, Piscataway, NJ, 2001), p. 2353.

[13] J. M. Seok, M. Chung, H. S. Kang, J. H. Han, and J. Hong, in Proceedings of IPAC2016, Busan, Korea (JACoW, 2016), p. 1054.

[14] S. Bettoni, P. Craievic, A. A. Lutman, and M. Pedrozzi, Temporal profile measurements of relativistic electron bunch based on wakefield generation, Phys. Rev. Accel. Beams 19, 021304 (2016).

[15] A. Novokhatski, A. Brachmann, M. D. Forno, V. Dolgashev, A. S. Fisher, M. Guetg, Z. Huang, R. Iverson, P. Krejcik, A. Lutman et al., in Proceedings of IPAC2016, Busan, Korea (JACoW, 2016), p. 817.

[16] M. W. Guetg, K. L. F. Bane, A. Brachmann, A. S. Fisher, M. A. Harrison, Z. Huang, R. Iverson, P. Krejcik, A. A. Lutman, T. J. Maxwell et al., in Proceedings of IPAC2016, Busan, Korea (JACoW, 2016), p. 809.

[17] J. Zemella, K. Bane, A. Fisher, M. Guetg, Z. Huang, R. Iverson, P. Krejcik, A. Lutman, T. Maxwell, A. Novokhatski et al., Measurements of wake-induced electron beam deflection in a dechirper at the Linac Coherent Light Source, Phys. Rev. Accel. Beams 20, 104403 (2017).

[18] C. Lu, F. Fu, T. Jiang, S. Liu, L. Shi, R. Wang, L. Zhao, P. Zhu, Z. Zhang, and D. Xiang, Time-resolved measurement of quadrupole wakefields in corrugated structures, Phys. Rev. Accel. Beams 19, 020706 (2016).

[19] G. Stupakov, Lecture notes on classical mechanics and electromagnetism in accelerator physics (2015), http://laser .lbl.gov/ gpenn/uspas2016/lectures_2011.pdf.
[20] P. B. Wilson, Report No. SLAC-PUB-4547, 1989.

[21] G. Burt, Cockcroft lectures: Deflecting cavities and crab cavities (2010), http://indico.hep.manchester.ac.uk/getFile .py/access? contribId=1\&resId=0\&materialId=slides\&confId= 1943.

[22] K. Bane and G. Stupakov, Impedance of a rectangular beam tube with small corrugations, Phys. Rev. ST Accel. Beams 6, 024401 (2003).

[23] G. Stupakov and K. Bane, Surface impedance formalism for a metallic beam pipe with small corrugations, Phys. Rev. ST Accel. Beams 15, 124401 (2012).

[24] H. S. Kang and J. Hong, in Proceedings of FEL2014, Basel, Switzerland (JACoW, 2014), p. 593.

[25] K. Flöttmann, ASTRA: A Space Charge Tracking Algorithm, http://www.desy.de/ mpyflo/.

[26] M. Borland, APS Technical Report No. LS-287, 2000.

[27] C. Behrens, F.-J. Decker, Y. Ding, V. A. Dolgashev, J. Frisch, Z. Huang, P. Krejcik, H. Loos, A. Lutman, T. Maxwell et al., Few-femtosecond time-resolved measurements of X-ray free-electron lasers, Nat. Commun. 5, 3762 (2014).

[28] J. W. Wang, S. G. Tantawi, C. Xu, M. Franzi, P. Krejcik, G. Bowden, S. Condamoor, Y. Ding, V. Dolgashev, J. Eichner et al., Development for a supercompact X-band pulse compression system and its application at SLAC, Phys. Rev. Accel. Beams 20, 110401 (2017).

[29] S.-Y. Kim, J.-M. Seok, K.-J. Moon, M. Chung, H.-S. Kang, C.-H. Kim, J. Hong, J.-H. Han, and J. Thangaraj, in Proceedings of FEL2015, Daejeon, Korea (JACoW, 2015), p. 694. 